\title{
Human-Oriented IoT-Based Interfaces for Multimodal Visual Analytics Systems
}

\author{
K.V. Ryabinin ${ }^{1, A}$, K.I. Belousov²,A, S.I. Chuprina3,A, N.L. Zelyanskaya4,A \\ Perm State University \\ ${ }^{1}$ ORCID: 00oo-0002-8353-7641, kostya.ryabinin@gmail.com \\ 2 ORCID: 0000-0003-4447-1288, belousovki@gmail.com \\ 3 ORCID: 0000-0002-2103-3771, chuprinas@inbox.ru \\ 4 ORCID: 0000-0002-5727-6919, zelyanskaya@gmail.com
}

\begin{abstract}
This paper describes an approach to use modern programmable microelectronics and ontology engineering to create custom hardware human-machine interfaces for solving particular visual analytics tasks. The idea of these special interfaces is to involve additional modalities like motor or haptic into the analytics process to improve its quality and speed. We propose using tangible user interfaces built upon the Internet of Things technologies to present the visual analytics system as a cyber-physical one, melting together the real and virtual environments. To automate the creation of such an alloy we suggest a unified approach of composing the firmware for the interface hardware device and corresponding drivers for the computer; of calibration the device' sensors; of testing and debugging the communication between the interface device and the application it is supposed to steer; and of solving visual analytics tasks using the device created. All the mentioned steps are supported by the highlevel built-in mechanisms of SciVi visual analytics platform we created during the previous research and improved in the current work. Data flow diagrams are used to visually describe the data preprocessing and rendering, as well as the ways hardware interface affects them. Ontology engineering is used to ensure flexibility and extensibility of the entire platform combined with the semantic power of its individual blocks: the behavior of SciVi is fully governed by underlaying ontologies, which describe supported data formats and types, filters, rendering mechanisms, supported electronic components of the hardware interfaces, the ways to program them and to communicate with them.

The proposed methods and means are tested by solving real-world visual analytics task of automated identification of the relationship between native speakers' psychological characteristics and their verbal behavior.
\end{abstract}

Keywords: visual analytics, Internet of Things, human-machine interface, ontology engineering, language semantics, multimodal analytics. 


\section{Introduction}

Technologies of the Internet of Things (IoT) [1] together with the corresponding edge [2] and fog [3] computing techniques enable rapid development of so-called cyber-physical systems, which are the alloy of the real and the virtual worlds and assume the computation power is incorporated and distributed within real-world everyday objects [4]. The key paradigm of human-machine interaction within cyberphysical systems is tangible user interface (TUI), proposed by Hiroshi Ishii in 1997 [5]. TUI assumes interaction with virtual objects through their physical "avatars" - real-world objects, which are not universal for the entire virtual world (like traditional controls, such as mouse or keyboard), but are close in shape, meaning and distinctive features to their virtual prototypes. Moreover, these physical objects often share some other functions, which are different from steering the virtual objects: they can be some real-world tools, pieces of interior, etc.

The nature of TUI implies multimodal interplay with the human that is far beyond traditional pushing of the buttons. First of all, haptic channel is employed as an addition to traditional audio and visual channels. This is especially important for the visually impaired people providing them with wide range of possibilities to communicate with the virtual environment. As per the task solved, other modalities can be combined with the haptics, for example, spatial gestures.

In our opinion, the multimodal nature of cyber-physical systems opens up the wide opportunities for humans in the field of complex data analytics. TUI for visual analytics systems enable expert to efficiently utilize his / her perceptive and cognitive mechanisms to understanding the features of the analyzed objects and thereby speed up the process and increase the quality of analysis.

Cyber-physical interaction of the human with the visual analytics system can be denoted as "preceptive-cognitive interface" (PCI). PCI is an ergonomic multimodal interface customized for the particular analytics task involving the human sensorymotor sphere and contributing in the speed and quality of analysis.

The aim of this work is to develop the concept of perceptive-cognitive interface for multimodal analytics tasks, as well as to describe the implementation of the appropriate software and hardware for confirming in practice the viability of the proposed concept.

\section{Perceptive-Cognitive Interface Concept}

The creation of perceptive-cognitive interfaces is based on the characteristics of the human psyche, which is capable of processing information transmitted by organs of sensory perception and harmonizing these heterogeneous multimodal signals to form a holistic picture of the world. Moreover, most of the understood and consciously processed information is transmitted in the process of verbal communication, which is mainly carried out using natural language through audio or visual channels. However, the ability of the psyche to structure information obtained through different channels of perception means that the other modes of sensory perception available to human also have great potential for understanding and cognition. Language semantics is closely related to the sensorimotor sphere of human. This is evidenced by research area of neuroscience and cognitive psychology (the socalled Embodiment Theories - theories involving the consideration of human consciousness in relation to physical environmental factors) [6-8]. The researchers have identified patterns between pronouncing words with the food semantics and salivation [9], reactions of the expansion or contraction of the pupil to words conveying 
darkness and brightness [10], manifestations of motor activity as a reaction to words with the meaning of actions [11], etc. In addition, it was found that the words associated with the activation of the sensorimotor experience consistent with the semantics of the word contribute to the understanding of messages, and, on the contrary, the words with the semantics of the contrasting (uncoordinated) sensorimotor experience hinder the understanding of verbal information.

Words (considered in context), which have several sensorimotor codes, are more easily recognized [12]. This finds practical application in the learning process [13] and in search technologies for multimodal (visual and audio) content [14]. The interconnection of words of abstract semantics with the sensorimotor sphere is described differently in the context of Embodiment Theories: either through the theory of conceptual metaphor introduced by G. Lakoff [8], or through the idea of a closer connection between abstract words and the emotional sphere [15].

The idea of perceptive-cognitive interfaces is based on Embodiment Theories and technological possibilities of using multimodal channels for the transmission of natural language information. From a technological point of view, this possibility is provided by a system of sensors used to activate text fragments of a certain sensory (sensorimotor) semantics. To activate, the semantics of sensors must be connected with the semantics of language content using a common formal model, which can be an ontology that links the levels of possible types of sensors (temperature, sound, light, movement in space - motility - and others) with the values of the sensors (color white, black, etc.; moving in space - up, down, right, left, etc.) and the names of semantic fields consisting of the meanings of words/phrases with perceptual semantics. Here, the semantic field is understood as "the totality of linguistic units, combined by a common content and reflecting the conceptual, substantive or functional similarity of the designated phenomena" [16].

The perceptive-cognitive interface in multimodal analytics systems allows the expert to use his / her perceptive-cognitive experience to find the pertinent information. The search strategy is based on the general hypothesis, in which proving process numerous operational hypotheses are used (for example, "Are there any messages in the analyzed data in which <condition>”). Operational hypotheses arise due to the knowledge gained about the relationship of individual modal values with each other and their regular presence in texts, i.e. their semantic standardization. In this context, the expert's perceptive-cognitive experience and operational hypotheses become part of the human-machine interface.

The Fig. 1 shows a fragment of the PCI ontological model for spatial movements' sensors (for a sake of simplicity, only four types of movement are presented: up, down, right and left). This ontology is used for ontologically controlled solution of the problem of determining the type of personality of a native speaker depending on the use of words with certain perceptual semantics in the framework of the state research project of Perm State University for 2017-2019, project No. 34.1505.2017/4.6 "Verbal and nonverbal behavior of a social network user: socio-cognitive modeling using machine learning methods and geoinformation technologies". 


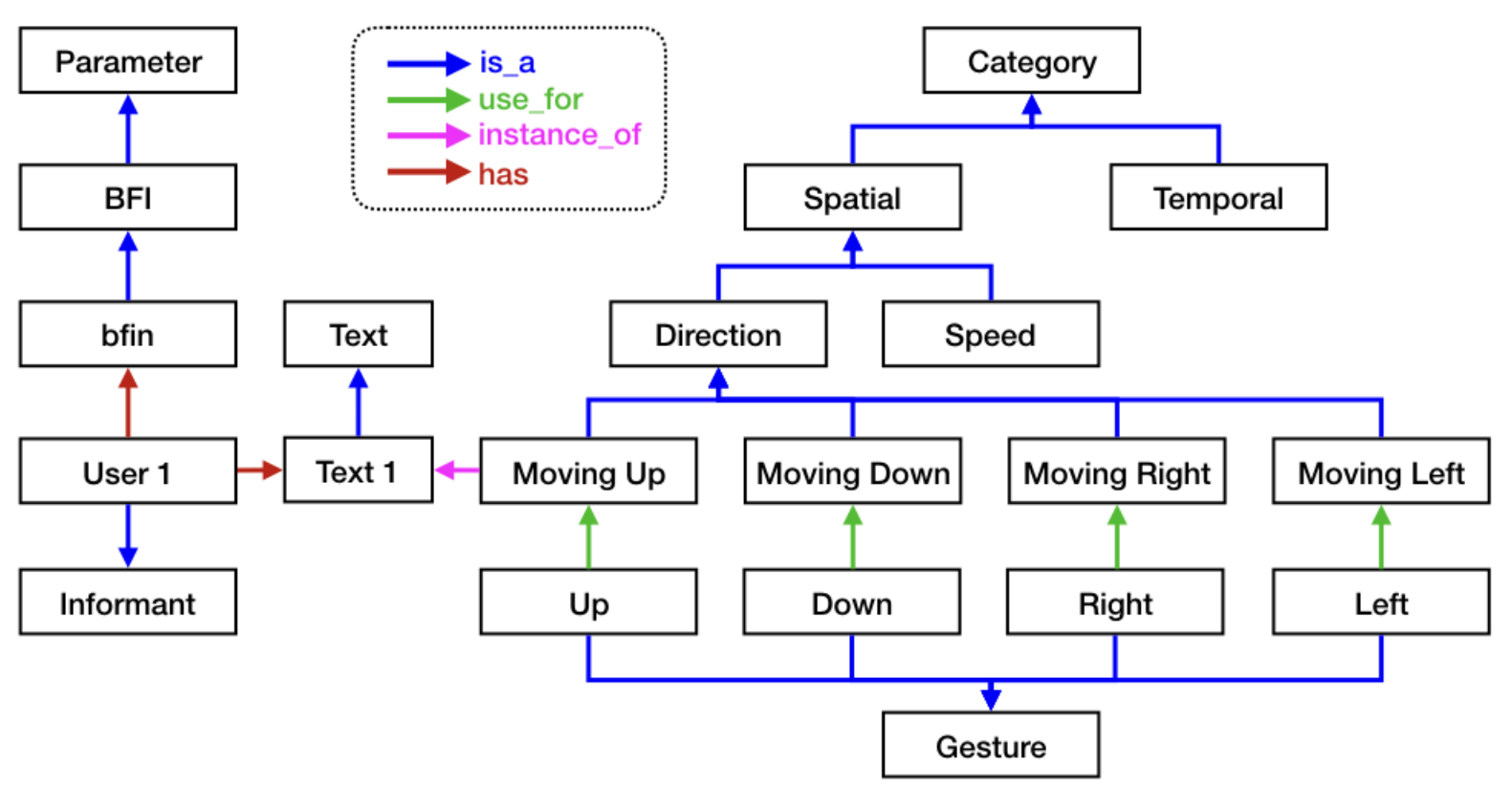

Fig. 1. A fragment of perceptive-cognitive interface ontology.

In the above fragment, the categorical semantics (Category) of the verbal messages is represented by "Spatial" and "Temporal" sub-categories. Spatiality, in its turn, is represented by the "Direction" and "Speed" of movement. The direction of movement is realized by means of antonymic pairs: "Moving Up" and "Moving Down", "Moving Right" and "Moving Left". Note that in this case we take into account only the semantics of messages (for example, "he raised his hands above his head", "look in the lower left corner of the screen", etc.). The semantics implemented in messages is directly related to the detectable gestures "Up", "Down", "Left" and "Right", which, in turn, can be used to control the process of visual analysis of the messages' semantics. For example, when using PCI gesture "Up", the content that has the semantics of moving up should be filtered ("Moving Up").

The instance "Text 1 ", is on the one hand a representative of the message class ("Text"), and on the other hand belongs to a specific author ("User 1"). "User 1", in turn, is an informant ("Informant"), who has some texts created by him / her and a set of parameters ("Parameter"), for example, the psychological parameters of the large five - factor personality questionnaire BFI (Big Five Inventory) and its values, for example, "bfin" - the severity of neuroticism: character traits predisposing to the experience of negative emotions [17].

Thus, the ontological model allows using gesture to filter content in accordance with the semantics of the gesture used, and then analyze the statistics of the parameters of personal characteristics of the authors of texts, in which this semantics is expressed. For example, if we want to know what percentage of informants with high rates of neuroticism refer in their statements to the semantics of moving up, it is enough for us to make a hand gesture "Up" within PCI.

The presented ontological model is implemented in the information system Semograph. This system is intended to automate the process of text data analyzing, creating the corpora, conducting and interpreting the results of psycholinguistic, sociolinguistic, and the like experiments, to create the classifiers and thesauri of subject areas, to construct the models and other tasks that arise during the analysis of text content $[18]$. 
To solve the problem of connecting gestures with message semantics, in Semograph we created a hierarchical classifier, which cells contain semantic fields, and the markup procedure is designated as field analysis (see. Fig. 2). The markup of the messages with spatial semantics was carried out by two experts. During the classification process all experts developed a concerted position on controversial issues.

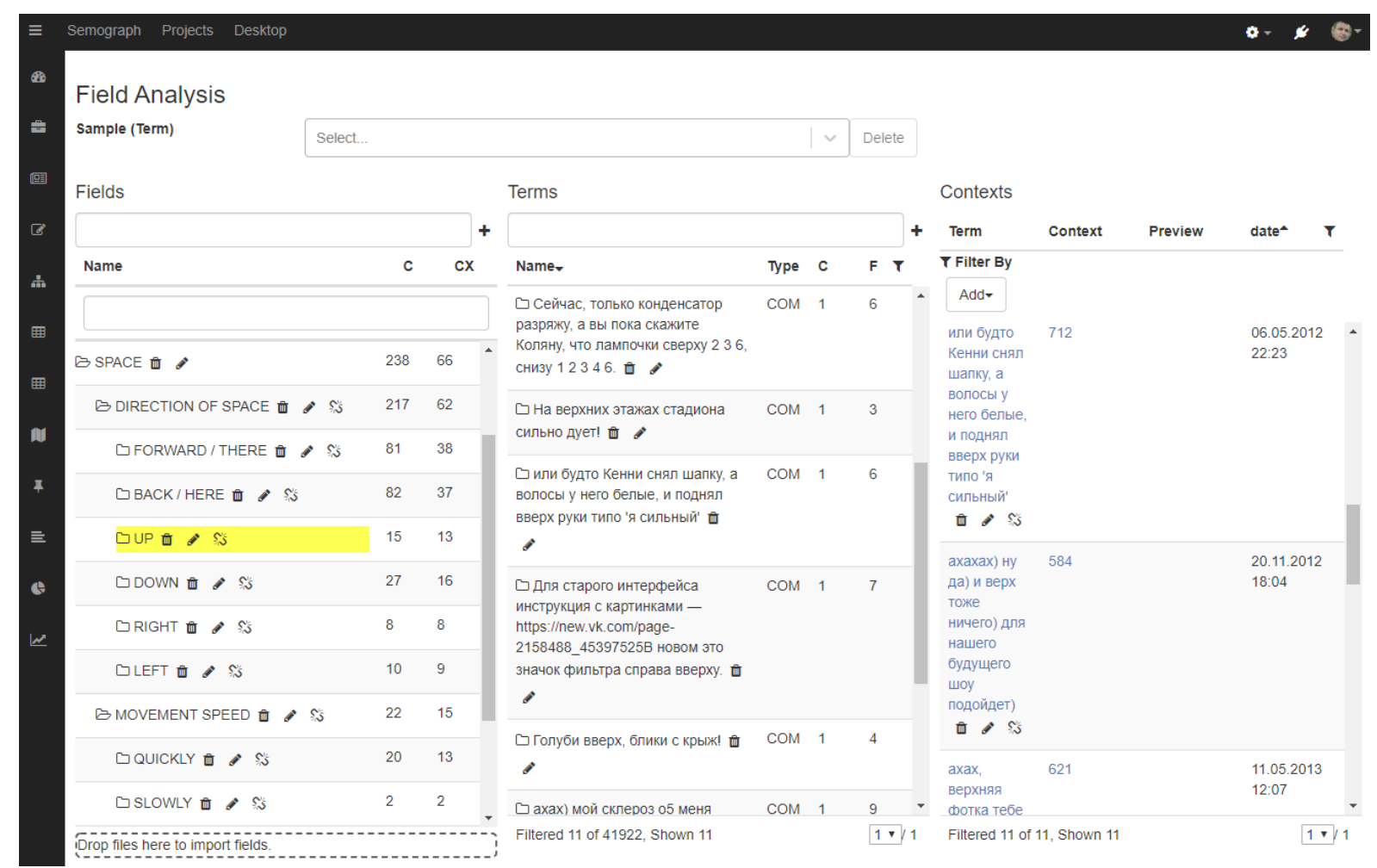

Fig. 2. Screenshot of the field analysis window that classifies user comments.

In the Fig. 2 it can be seen that the field analysis window consists of three areas: "Fields", "Terms" and "Contexts". The left column of "Fields" shows semantic fields. "Terms" (comments of the social network users) are filtered by the UP field (only comments that are included in this field are displayed). In the "Contexts" column we can see the same comments with their additional parameters, including links to the contexts in which they occur. The "Terms" column reflects the frequency of using comments in the entire reaction corpus (column " $\mathrm{C}$ ") and the number of occurrences of this unit in semantic fields (column " $F$ ").

An important feature of the presented model is the hierarchical organization of semantic fields and their potential extensibility. For example, each field with spatial semantics can be divided into three subfields: the first subfield consists of lexical units with an explicit expression of sensual semantics, the second subfield is composed of lexical units with an implicit expression of sensual semantics, and the third one consists of lexical units associated with this semantics indirectly, for example, using metaphorical / metonymic transfers, etymology, etc. This approach allows us, on the one hand, to expand significantly the repertoire of lexical units covered by the presented model, and on the other hand, to choose the level of semantic complexity appropriate for the expert.

The hardware part of PCI in this case is a glove-style device that detects spatial gestures by using inertial measurement unit (angular position detector) MPU6050 and programmable microcontroller ESP8266. Software part of PCI is SciVi visual analytics platform [19] that interprets the gestures and treats them as semantic filters for 
the data visualized as a graph. This graph demonstrates the relationships between the verbal behavior of people and their psychological characteristics revealed using machine learning methods [20-22].

\section{Perceptive-Cognitive Interface within SciVi Visual Analytics Platform}

The PCI is distinct from the traditional human-machine interface (like mouse or keyboard) by its specialization: its hardware part is customized to suit the particular visual analytics task. In this sense it is close to the GUI, which appearance may vary from task to task. However, PCI involves changes both in virtual and in physical part of the interface. This, in turn, requires high-level tools and high-level hardware and software building blocks to enable an expert to assemble a custom PCI without having deep skills in programming or electronics. SciVi platform provides corresponding mechanisms to automate customizations in the software part of PCI.

We propose the following life cycle of PCI within SciVi:

1. Designing the PCI.

2. Assembling the hardware part of PCI.

3. Writing and installing the software part of PCI (firmware for the device and driver for the computer).

4. Calibration of the device' sensors.

5. Testing and debugging of the communication between PCI and application it is supposed to steer.

6. Solving analytics tasks with PCI.

Traditionally, each stage of this life cycle is supported by different, often unrelated instruments. In this work we propose to utilize SciVi visual analytics platform [19] as a unified approach for PCI creation and usage. SciVi includes high-level flexible adaptation and customization mechanisms governed by ontologies. It leverages communication with different data sources to obtain data for analytics, provides mechanisms to declare preprocessing (filtering) and visualization algorithms as well as supports device firmware generation. Thanks to this, SciVi can help to fulfill stages 3-6 in a uniform way making the PCI creation available for experts without advanced software engineering skills.

Designing and assembling stages cannot yet be automated, but the electronic components ontology of SciVi [19] has, among others, the recommendation function, as it contains a description of various microcontrollers, sensors, actuators and commutators, as well as methods of their interactions. It can be treated as a guideline, which components fit together and how they should be interconnected.

The firmware generation mechanism included with SciVi operates on the basis of the electronic components' ontology indicated above [23]. The logic of the PCI, as well as the visualization and analysis algorithms, are declared using a data flow diagram (DFD), composed by the user within built-in high-level editor. This altogether allows almost complete automation of the PCI software part development.

Fig. 3 presents the basic concept of SciVi usage as a platform for PCI-powered visual analytics. 


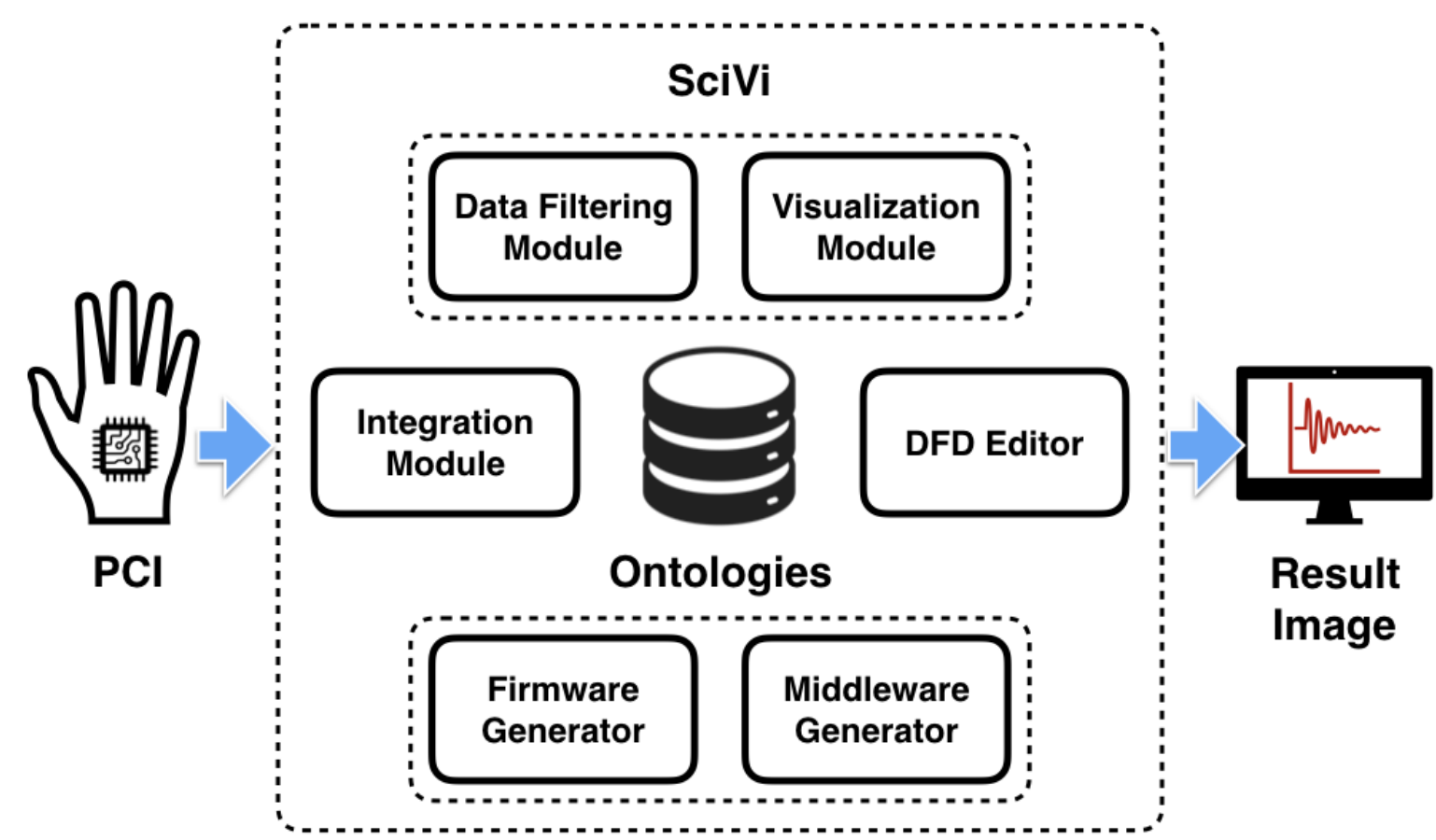

Fig. 3. PCI in SciVi: blue arrows denote the main direction of both data and control flows.

Fig. 4 demonstrates the DFD describing firmware for ESP8266 microcontroller used in the glove PCI that has been assembled during the current work.

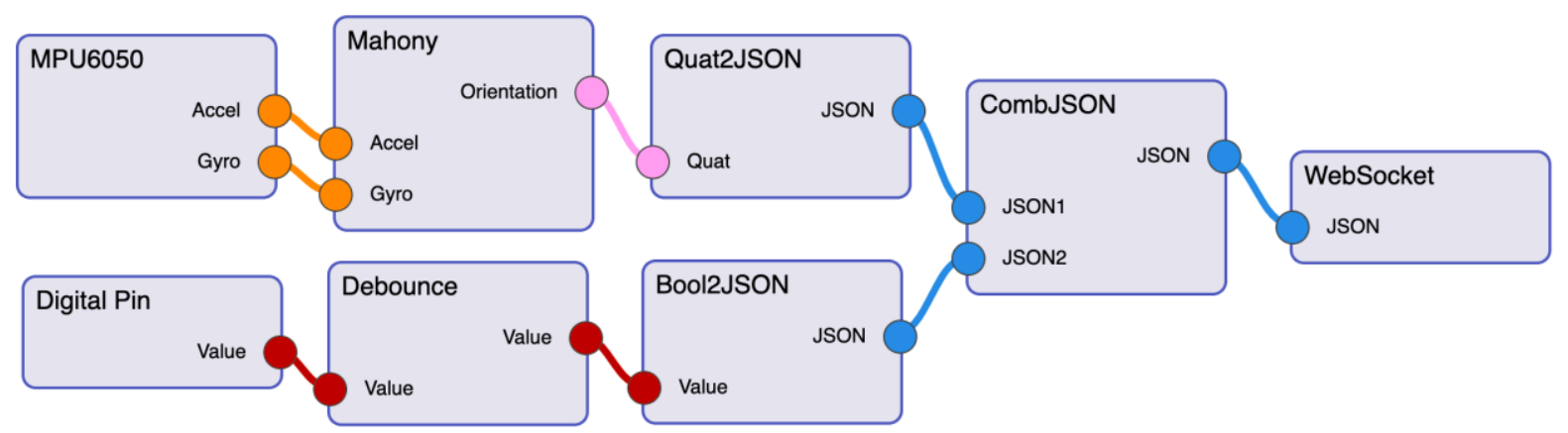

Fig. 4. Data flow diagram describing device firmware.

The data sources in this case are the inertial measurement unit (IMU) MPU6050, supplying acceleration and angular velocity of the glove and digital pin, connected to the button pushed when index finger is bent. The IMU data are passed to Mahony filter [24] that transforms them into quaternion describing the glove's orientation. The boolean signal from digital pin is debounced to reduce the random noise inevitably appearing when button is pushed. Then, both orientation quaternion and finger bending flag are serialized in JSON format, combined into single message and transmitted via WebSocket over the WiFi.

According to the signal message format (defined by serialization and transmission nodes), ontological profile of the device is automatically created. This profile describes the output data of the device, which should be used as control signals during the visual analytics process. The ontological profile enables to generate the node that represents the device as a control signal source as it is schematically shown in the Fig. 5 . 


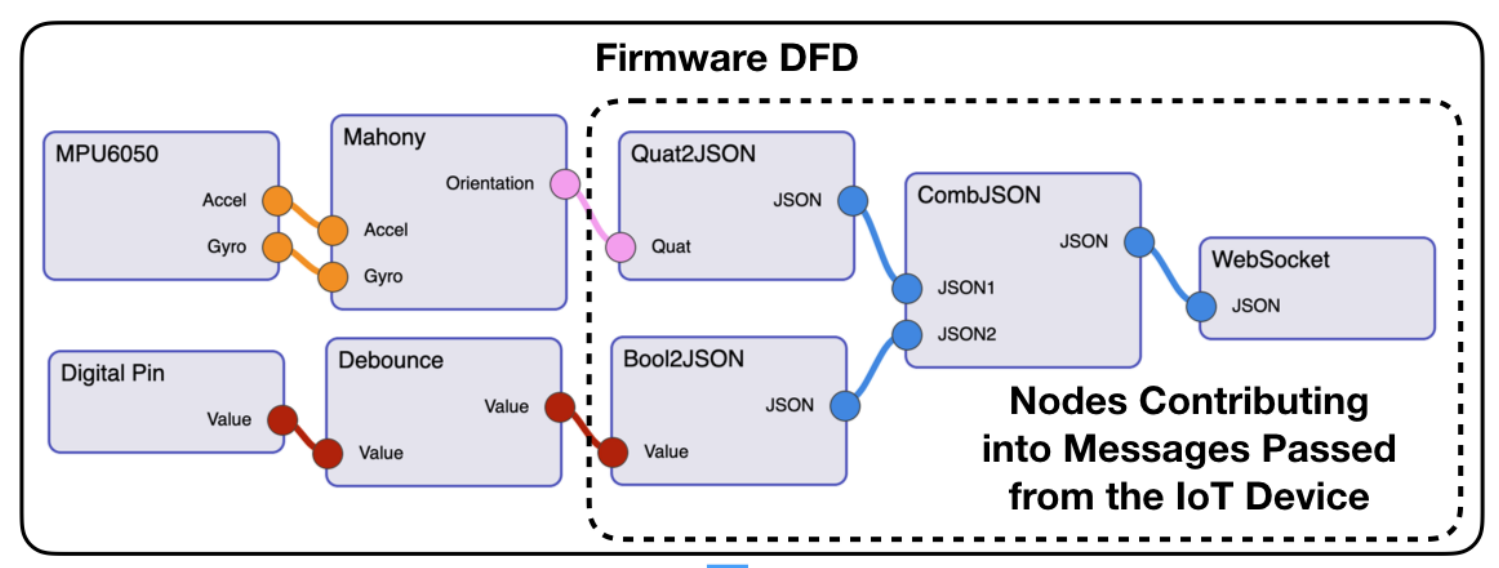

\section{Generation}
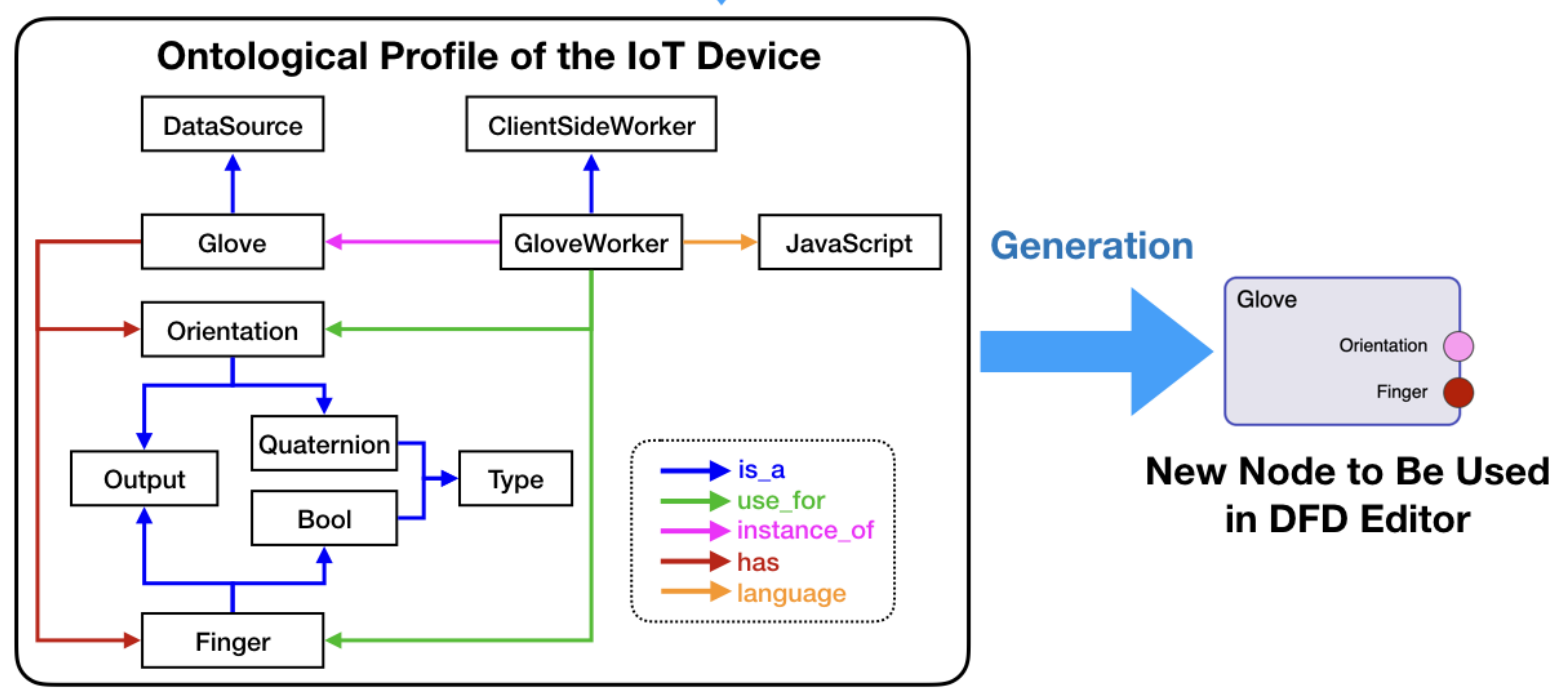

Fig. 5. Generation of the device ontological profile.

Calibration of the PCI sensors can be simplified by means of visual monitoring of the control signals obtained from the device. SciVi DFD editor provides fast and flexible way of choosing the most observable and informative form of data displaying, and thereby helps to efficiently estimate the sensor measurement errors [25]. The graphical user interface generator within SciVi allows to incorporate feedback widgets into the visualization view. These widgets can be used to set up calibration parameters and transfer them back to the device. For example, threshold values can be tuned at runtime to compensate the sensor errors and noise [25].

The debug of the interaction between PCI and particular graphical scene visualized by SciVi is also based on modifying the DFD: the user can try out different combinations of rendering algorithms and semantic filters for the data, searching for the most convenient and observable variants. Thereby the one can rapidly examine, if the particular PCI suites the needs of current visual analytics task, or it has to be improved. Ontology driven functioning of SciVi simplifies the extending of the platform's capabilities: if the new semantic filters or rendering mechanisms are required for the particular visual analytics task, they can be added without changing the source code of the SciVi core. Thereby, SciVi can be tuned for solving the wide range of visualization and analytics problems, involving both traditional graphical user interfaces and PCI. 
Fig. 6 demonstrates DFD that describes the mapping of the different gestures to the language semantics. The "Glove" node denotes communication with the glove PCI. The connection is established automatically; technical details of data transmission needed for this connection are described in the ontological profile of the glove device. The nodes "Getsure Up", "Gesture Down", "Gesture Left" and "Gesture Right" describe detectors of corresponding gestures. The key DFD element is the node "Classifier" depicting the filter, that actually maps gestures to the language semantics according to the ontology, that has been shown in the Fig. 1. Under the hood this filter generates ontology driven algorithm for selecting the data, which semantics matches the gesture detected, and transmits this algorithm to the graph view represented by "BFI Graph" node. The "BFI Graph" node denotes the visualization type of the data and the data itself. In this example, for the sake of DFD simplicity, the data set is tied to the visual object as a parameter accessible through the node's settings. The rendering result is shown in the Fig. 7. According to the DFD described above, data filtering in a circular graph [22] is controlled by the glove PCI.

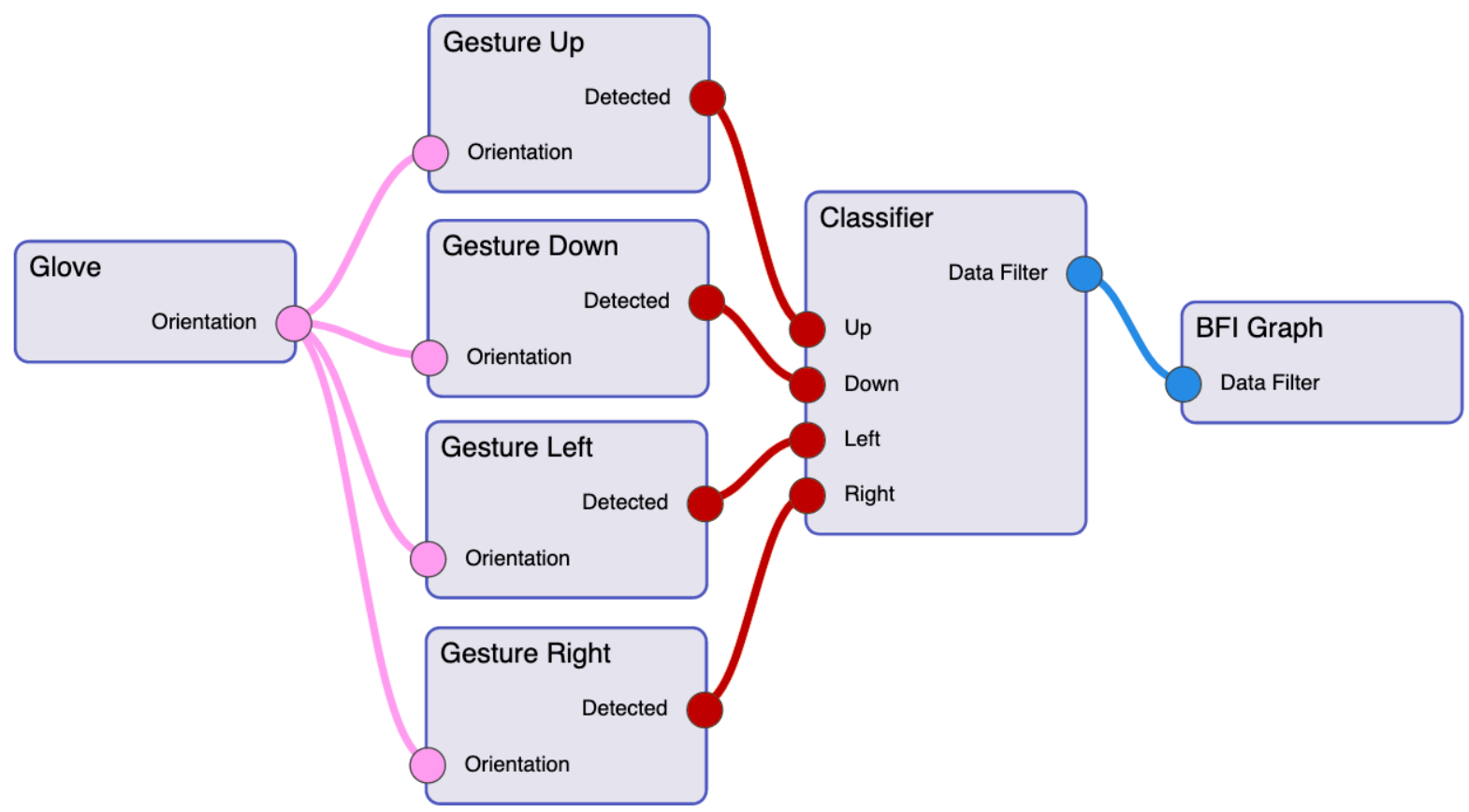

Fig. 6. Data flow diagram describing gesture-based visual analytics task. 


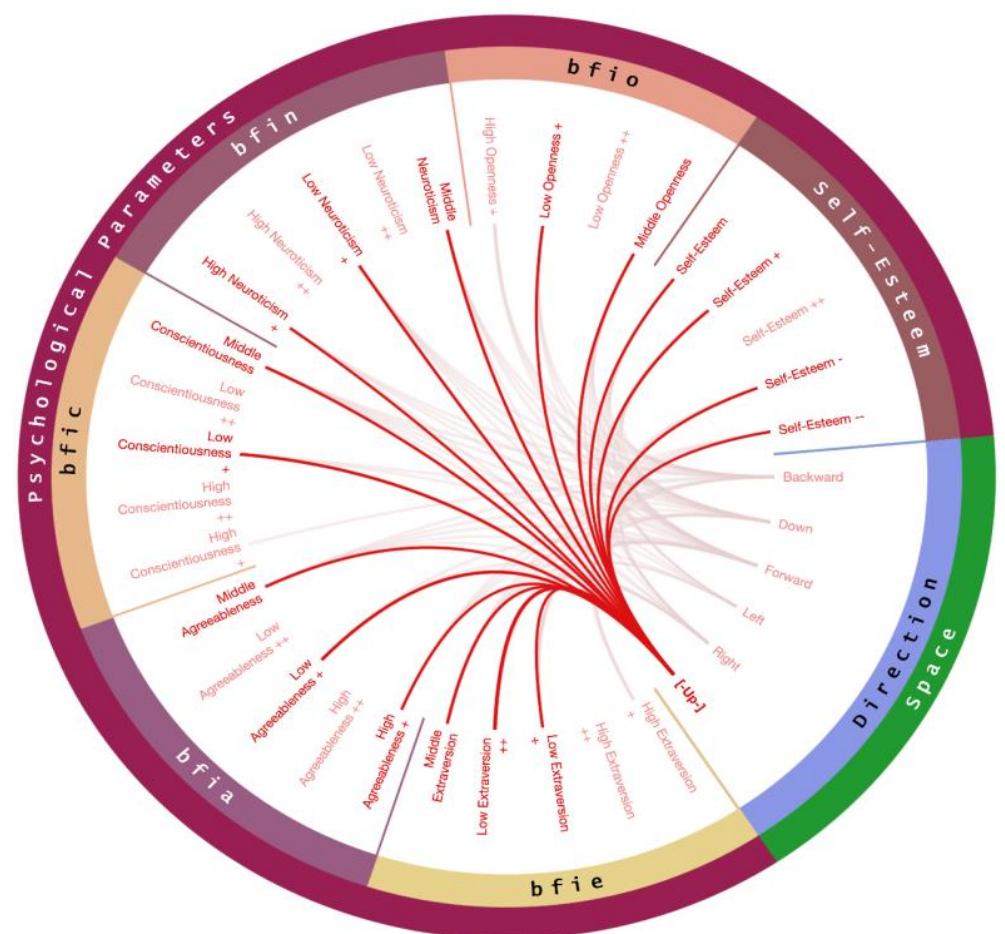

Fig. 7. Circular graph rendered according to the data flow diagram from the Fig. 6.

\section{Conclusion}

Our previous research allowed to transform SciVi scientific visualization system into the feature-packed visual analytics platform. In the current work we integrated methods and means of ontology engineering with IoT technologies to enrich SciVi with hardware perceptive-cognitive human-machine interface. This is a first step towards multimodal analytics systems, involving both visual and sensorimotor perceptive channels of the expert. Technique proposed were used to solve a practical problem of analyzing the dependencies between psychological parameters and verbal behavior of social network users. According to the proposed approach a PCI was developed that enables to control the data search and filtering mechanisms with spatial gestures, associated with corresponding spatial semantics. Gestures are detected with special glove manipulator that operates on the basis of inertial measurement unit. Presented ontology driven classifier maps these gestures to the data selection algorithms, which build up a semantic filter for the data being visualized. This, in turn, speeds up the analysis process allowing the expert do find the relevant data just by the single gesture without any textual queries, sliders dragging, etc.

In the future, it is planned to expand the range of supported PCI modalities with a haptic channel, using various types of sensors and various methods of their integration into sensor networks based on the principles of IoT technologies. In addition, it should be examined how tight the expert's perceptual and cognitive experience is converged with PCI, because in the process of multimodal analytical activity the use of this type of interface can qualitatively transform the expert's perceptual-cognitive experience due to the possible formation of additional neural connections between visual, auditory, motor, and other centers. These studies will be carried out using the capabilities of the 128-channel BE Plus LTM neurovisor. In the future, this can significantly improve the methods and means of automated transformation of machineto-machine IoT-systems into human-centric ones. 


\section{Acknowledgements}

The reported study is partially supported by Ministry of Education and Science of the Russian Federation, State Assignment No. 34.1505.2017/4.6 (Research Project of Perm State University, 2017-2019).

\section{References}

1. Rose, K., Eldridge, S., Chapin, L. The Internet of Things: an Overview [Electronic Resource] // The Internet Society (ISOC). - 2015. URL: https://www.internetsociety.org/resources/doc/2015/iot-overview (last accessed 09.10.2019).

2. Khan, W., Ahmed, E., Hakak, S., Yaqoob, I., Ahmed, A. Edge computing: A survey // Future Generation Computer Systems. - Elsevier, 2019. - Vol. 97. - PP. 219235. DOI: 10.1016/j.future.2019.02.050.

3. Zhang, P., Zhou, M., Fortino, G. Security and trust issues in Fog computing: A survey // Future Generation Computer Systems. - Elsevier, 2018. - V. 88. - PP. 16-27. DOI: 10.1016/j.future.2018.05.008.

4. Sanfelice, R. Analysis and Design of Cyber-Physical Systems. A Hybrid Control Systems Approach // Cyber-Physical Systems: From Theory to Practice / Rawat, D., Rodrigues, J., Stojmenovic, I. - CRC Press, 2015. - PP. 3-31. DOI: 10.1201/b19290-3.

5. Ishii, H., Ullmer, B. Tangible Bits: Towards Seamless Interfaces Between People, Bits and Atoms // CHI '97 Proceedings of the ACM SIGCHI Conference on Human Factors in Computing Systems. - ACM, 1997. - PP. 234-241. DOI: 10.1145/258549.258715.

6. Barsalou, L. Perceptual Symbol Systems // Behavioral and Brain Sciences. 1999. - Vol. 22. - PP. 577-609.

7. Pulvermüller, F. Words in the Brain's Language // Behavioral and Brain Sciences. - 1999. - Vol. 22. - PP. 253-279.

8. Gallese, V., Lakoff, G. The Brain Concepts: the Role of the Sensorymotor System in Conceptual Structure // Cognitive Neuropsychology. - 2005. - Vol. 22, I. 3. PP. 455-479. DOI: 10.1080/02643290442000310.

9. Staats, A., Hammond, O. Natural Words as Physiological Conditioned Stimuli: Food-Word-Elicited Salivation and Deprivation Effects // Journal of Experimental Psychology. - 1972. - Vol. 96, I. 1. - PP. 206-208. DOI: 10.1037/hoo33508.

10. Laeng, B., Sulutvedt, U. The Eye Pupil Adjusts to Imaginary Light // Psychological Science. - 2013. - Vol. 25, I. 1. - PP. 188-197. DOI: 10.1177/0956797613503556.

11. Aravena, P., Delevoye-Turrell, Y., Deprez, V., Cheylus, A., Paulignan, Y., Frak, V., Nazir, T. Grip Force Reveals the Context Sensitivity of Language-Induced Motor Activity during "Action Words" Processing: Evidence from Sentential Negation // PLoS ONE. - 2012. - Vol. 7, I. 12. DOI: 10.1371/journal.pone.0050287.

12. Hoffman, P., Lambon Ralph, M. Shapes, Scents and Sounds: Quantifying the Full Multi-Sensory Basis of Conceptual Knowledge // Neuropsychologia. - Elsevier, 2013. - Vol. 51, I. 1. - PP. 14-25. DOI: 10.1016/j.neuropsychologia.2012.11.009.

13. Lockwood, G., Hagoort, P., Dingemanse, M. How Iconicity Helps People Learn New Words: Neural Correlates and Individual Differences in Sound-Symbolic Bootstrapping // Collabra: Psychology. - 2016. - Vol. 2, I. 1. - PP. 1-15. DOI: 10.1525/collabra.42. 
14. Chang, Sh.-F., Ellis, D., Jiang, W., Lee, K., Yanagawa, A., Loui, A., Luo, J. LargeScale Multimodal Semantic Concept Detection for Consumer Video // Multimedia Information Retrieval. - 2007. - P. 255-264. DOI: 10.1145/1290082.1290118.

15. Meteyard, L., Cuadrado, S., Bahrami, B., Vigliocco, G. Coming of Age: a Review of Embodiment and the Neuroscience of Semantics // Cortex. - Elsevier, 2012. Vol. 48, I. 7. - PP. 788-804. DOI: 10.1016/j.cortex.2010.11.002.

16. Kuznetsov, A. Field [in Russian] // Linguistics. Great Academic Dictionary. Sec. Ed. - Great Russian Encyclopedia, 1998. - 685 p.

17. Shchebetenko, S. Reflexive Characteristic Adaptations Explain Sex Differences in the Big Five: but not in Neuroticism // Personality and Individual Differences. 2017. - Vol. 111. - PP. 153-156. DOI: 10.1016/j.paid.2017.02.013.

18. Belousov, K., Erofeeva, E., Leshchenko, Y., Baranov, D. "Semograph" Information System as a Framework for Network-Based Science and Education // Smart Education and e-Learning. - Springer, 2017. - PP. 263-272. DOI: 10.1007/978-3319-59451-4_26.

19. Ryabinin, K., Chuprina, S., Kolesnik, M. Calibration and Monitoring of IoT Devices by Means of Embedded Scientific Visualization Tools // Lecture Notes in Computer Science. - Springer, 2018. - Vol. 10861. - PP. 655-668. DOI: 10.1007/978-3-319-93701-4_52.

20. Ryabinin K.V., Belousov K.I., Chuprina S.I., Shchebetenko S.A., Permyakov S.S. Visual Analytics Tools for Systematic Exploration of Multi-Parameter Data of Social Web-Based Service Users // Scientific Visualization. - National Research Nuclear University "MEPhI", 2018. - Q. 3, Vol. 10, No. 4. - PP. 82-99. DOI: 10.26583/sv.10.4.07.

21. Ryabinin, K.V., Baranov, D.A., Belousov, K.I. Integration of Scientific Visualization Toolset SciVi with Information System Semograph // Proceedings of 27th International Conference GraphiCon 2017. - 2017. - PP. 138-141.

22. Ryabinin, K.V., Chuprina, S.I., Belousov, K.I., Permyakov, S.S. Visual analytics methods of the verbal behavior variability of social networks users depending on their individual psychological features // Proceedings of $28^{\text {th }}$ International Conference GraphiCon 2018. - 2018. - PP. 163-167.

23. Ryabinin, K., Chuprina, S., Belousov, K. Ontology-Driven Automation of IoTBased Human-Machine Interfaces Development // Lecture Notes in Computer Science. - Springer, 2019. - Vol. 11540. - PP. 110-124. DOI: 10.1007/978-3-03022750-0_9.

24. Mahony, R., Hamel, T., Pflimlin J. Nonlinear Complementary Filters on the Special Orthogonal Group // IEEE Transactions on Automatic Control. - IEEE, 2008. - Vol. 53, No. 5. - PP. 1203-1218. DOI: 10.1109/TAC.2008.923738.

25. Ryabinin, K., Chuprina, S. High-Level Toolset For Comprehensive Visual Data Analysis and Model Validation // Procedia Computer Science. - Elsevier, 2017. Vol. 108. - PP. 2090-2099. DOI: 10.1016/j.procs.2017.05.050. 